\title{
Programa de transferencia de conocimiento de la empresa a la universidad: una estrategia de comunicación para dar a conocer una marca industrial ${ }^{1 .}$
}

\author{
Knowledge Transfer Program Company A college : Communication Strategy to \\ give industrial know a brand.
}

\section{Vivian Andrea Montoya Ruiz²}

Marcela Nivia $\mathbf{F}^{3}$

Montoya R. Vivian A, Nivia F. Marcela. miradas Nº13 - 2015. ISSN: 0122 994X Págs 68 - 87

Recepción: Mayo 7 de 2015

Aprobación: Septiembre 24 de 2015

Publicación: Diciembre 17 de 2015

\section{Resumen}

El presente trabajo de investigación fue desarrollado con el fin de analizar los efectos que tiene la implementación de un programa de trasferencia de conocimiento sobre la estrategia de comunicación para dar a conocer la marca industrial ABB. Este trabajo abordó a los empleados de la empresa como agentes transmisores y a los estudiantes universitarios de ingeniería eléctrica como receptores de esta información.

Para la construcción, implementación y análisis del programa de transferencia, se revisó la percepción de los empleados de $\mathrm{ABB}$, el papel del área de Comunicaciones Corporativas y el nivel de conocimiento de la marca $\mathrm{ABB}$ por parte de los estudiantes de ingeniería. Los resultados obtenidos evidenciaron que un programa de transferencia de conocimiento puede convertirse en una estrategia de comunicación efectiva para dar a conocer la marca de empresas industriales, dando valor a los procesos comunicativos y aportando a la educación de los universitarios.

1 Trabajo de investigación original: Programa de transferencia de conocimiento de la multinacional Suiza ABB hacia los estudiantes de ingeniería eléctrica: una estrategia de comunicación para dar a conocer la marca. Caso de la multinacional Suiza ABB en Colombia. Universidad de la Sabana, Facultad de Comunicación Maestría en Comunicación Estratégica 2 Comunicadora Social y Periodista de la Universidad de la Sabana. Especialista en Gerencia Comercial de la Universidad de la Sabana Gerente de Comunicaciones Corporativas, ABB Colombia y Ecuador

Correo electrónico: vivian.montoya@co.abb.com

3 Comunicadora Social y Periodista de la Universidad de la Sabana. Especialista en Comunicación Organizacional, Universidad Javeriana Master's Degree, Marketing, Universidad Jorge Tadeo Lozano. Directora de tesis de grado de la Maestría en Comunicación Estratégica, Universidad de la Sabana Correo electrónico: adriana.nivia@unisabana.edu.co 
Como resultado, en un período de 12 meses, 10 empleados de ABB en Colombia lograron dar a conocer los aspectos de la marca $\mathrm{ABB}$ en 717 estudiantes de ingeniería de 5 universidades del país, que previamente no conocían esta marca.

\section{Palabras Clave: Conocimiento,} empleados, comunicación, estudiantes, estrategias.

\begin{abstract}
This research project was developed in order to analyze the effects of the implementation of a transfer knowledge program of the ABB Swiss Company to the electrical engineering students, over the communication strategy to publicize the ABB brand. As part of this research the employees were addressed as transmitter agents of the organization knowledge. In turn, it was proposed to achieve the electrical engineering students as recipients of this knowledge.
\end{abstract}

For the construction, implementation and analysis of the transference program, it was necessary to analyze the ABB employee's perception, the corporate communications strategies role, and the ABB brand's knowledge level from engineering students. The results showed that a transfer knowledge program can be an effective communication strategy to publicize the industrial company's brands, valuing communication processes and contributing to the college education.

As a result, over a 12 months period, 10 employees of $\mathrm{ABB}$ in Colombia were able to announce of the ABB brand's aspects over 717 engineering students from five universities in the country, most of whom did not know ABB's brand before.

Key Words: Knowledge, employees, communication, students, strategies.

\section{Introducción}

Las compañías altamente especializadas, tecnológicas e industriales cuyos productos $\mathrm{y}$ servicios se promocionan mediante estrategias enfocadas únicamente a sus clientes directos, tienen el gran reto de utilizar todos sus recursos para darse a conocer ante otros grupos de interés relevantes. Según Ries y Trout (2002) el posicionamiento de una marca consiste en concentrarse en una idea, o incluso en una palabra que defina a la compañía en las mentes de los consumidores. Tener una marca fuerte que identifique a la empresa da una ventaja competitiva relevante.

Con base en lo anterior, se planteó como problema de investigación que las grandes organizaciones industriales de carácter multinacional invierten grandes sumas dinero en investigación y desarrollo, lo que las hace poseedoras de valiosa información $\mathrm{y}$ conocimiento sobre innovación y tecnología, pero en algunas ocasiones, todo este conocimiento se queda al interior de la organización y sale convertido en un producto o servicio que llega directamente al cliente y no se aprovecha la oportunidad de contribuir al ámbito universitario y académico, donde sus estudiantes podrían ser en el futuro potenciales clientes de sus productos y servicios.

A raíz del problema identificado, este trabajo de investigación analizó el caso de la multinacional Suiza de ingeniería ABB, reconocida por el MIT Technology Review (2013) como una de las 50 empresas más innovadoras del mundo en la categoría especial de "las 50 empresas más disruptivas de mundo". El estudio abordó a la empresa $\mathrm{ABB}$ en Colombia, con el objetivo de identificar los efectos que tiene un programa de transferencia de conocimiento de esta empresa sobre la estrategia de comunicación para dar a conocer su marca, 
donde los empleados fueron contemplados como los transmisores del conocimiento y los estudiantes universitarios de ingeniería eléctrica fueron los receptores. La investigación se llevó a cabo del año 2012 al 2014 mediante el análisis de 234 empleados de ABB procedentes de ciudades como Bogotá, Medellín, Cali, Pereira, Barranquilla y Bucaramanga. A su vez se abordó a 717 estudiantes de las facultades de ingeniería eléctrica de instituciones de educación superior como la Universidad Distrital Francisco José de Caldas, la Universidad Nacional de Medellín, Universidad de Antioquia, Universidad Pontificia Bolivariana y la Universidad Militar.

Debido a la escasa información que existe sobre marketing industrial en comparación a los trabajos de investigación sobre marketing para empresas de productos y servicios de consumo masivo, toma relevancia la presente investigación ya que centra su estudio en una multinacional de ingeniería industrial como $\mathrm{ABB}, \mathrm{y}$ adicionalmente propone estrategias para mejorar su comunicación de marca en sus grupos de interés relevantes, aspecto que no se ha profundizado a nivel investigativo, de acuerdo a la información consultada.

La pregunta principal que suscitó la presente investigación fue ipuede un programa de transferencia de conocimiento liderado por los empleados de las empresas industriales hacia los estudiantes universitarios, convertirse en una estrategia de comunicación para dar a conocer la marca? Para dar solución al problema de investigación fue necesario abordar otras inquietudes como ¿qué tipo de conocimiento generan estas empresas industriales?; ¿Qué papel juegan los estudiantes universitarios para las empresas industriales? ¿Qué tipo de empleados dentro de la organización pueden ser los transmisores de ese conocimiento?, ¿Qué papel juega el área de Comunicaciones Corporativas en esta estrategia de comunicación para dar a conocer la marca?,¿cómo se debe estructurar un programa de transferencia de conocimiento para dar a conocer una marca industrial?; ¿Cuál es la percepción de los estudiantes universitarios frente a este tipo de estrategias?, ¿Cuáles son los efectos de un programa de transferencia de conocimiento en número de personas abordadas?

Con el objetivo de responder a todas las preguntas planteadas se aplicaron como instrumentos de investigación el análisis documental, análisis teórico, aplicación de encuestas, entrevistas y aplicación de modelos teóricos. Se abordaron algunos conceptos sobre las relaciones académico empresariales y el rol del conocimiento en estas relaciones.

Se analizó la literatura existente sobre programas de transferencia de conocimiento y sus objetivos dentro de las relaciones académico empresariales. Se presentaron también los conceptos de marketing industrial, empresas B2B, las características de las marcas y su rol en las estrategias de comunicación externa. Se analizó el papel de los estudiantes universitarios en las relaciones entre la empresa y la academia junto con el tipo de conocimiento que requieren estos estudiantes por parte de las empresas dependiendo de su carrera profesional.

Se analizó también, la percepción de los empleados de ABB acerca de las estrategias de comunicación que utiliza la organización para dar a conocer su marca y su interés de participar en estas estrategias de comunicación mediante la transferencia de sus conocimientos. Con la información encontrada se diseñó e implementó el programa donde los empleados de $\mathrm{ABB}$ 
fueron los transmisores y los estudiantes de ingeniería eléctrica de 5 universidades del país fueron los receptores del conocimiento. Para determinar los efectos de este programa sobre la estrategia de comunicación para dar a conocer la marca, se analizaron los resultados de una encuesta de percepción aplicada a los estudiantes que participaron y se revisaron algunos medios de comunicación como el Fan Page de Facebook y medios impresos y virtuales que fueron utilizados en el proceso de difusión del programa. Y finalmente se realizó una entrevista a un empleado que participó como promotor del programa de transferencia de conocimiento para conocer su percepción sobre esta iniciativa y de esta manera fue posible tener la visión completa de todos los grupos abordados en el estudio, antes y después de la implementación del programa.

Los resultados obtenidos en la investigación permitieron validar la hipótesis de que la transferencia del conocimiento de compañías multinacionales de ingeniería como $\mathrm{ABB}$ puede constituirse en una estrategia de comunicación efectiva para dar a conocer la marca, donde los empleados sean sus transmisores y los estudiantes de ingeniería sean sus receptores.

\section{Materiales y métodos}

\section{Diseño}

La presente investigación de carácter mixto (cualitativa y cuantitativa) que apunta a la compresión de la realidad como resultado de un proceso histórico de construcción a partir de las lógicas de sus protagonistas con una óptica interna y externa, y destacando su diversidad y particularidad (Galeano 2004), realizó un estudio de caso enfocado en la empresa multinacional Suiza de ingeniería $\mathrm{ABB}$ Colombia frente a la pertinencia de la creación de un programa de transferencia de conocimiento, por parte de sus empleados hacia los estudiantes universitarios, como una estrategia de comunicación para dar a conocer la marca.

Se utilizó la metodología de estudio de caso siguiendo los planeamientos de Smith y Zeithaml (1999) que lo entienden como un método que se ajusta adecuadamente a la intención de generar nuevos elementos en la investigación además de habilitar al investigador a ser sensible al carácter del caso y a estudiarlo como un proceso de desarrollo a través del tiempo.

En este sentido, el estudio de caso de $\mathrm{ABB}$ en Colombia, persiguió objetivos exploratorios que lograron contribuir a la construcción y desarrollo de perspectivas teóricas en torno a organizaciones como $\mathrm{ABB}$ y que además permitieron aproximarse a sus procesos de comunicación. Esta investigación exploratoria se enfocó en el análisis del problema para proporcionar conocimiento y entendimiento.

Para desarrollar la investigación se aplicó el siguiente esquema de familias, enfoques y técnicas de investigación diseñado por Hughes (2004) según la figura 1-1.

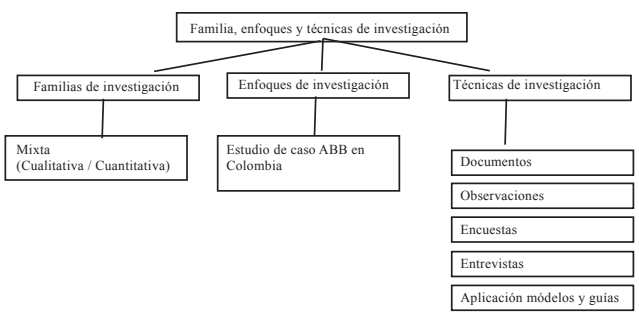

Figura 1-1: Esquema metodológico de investigación Fuente: Esquema metodológico para la investigación Hughes (2004) página 90.

\section{Unidades de análisis y entorno}

- Los empleados de las áreas de negocio de la multinacional Suiza ABB en Colombia en sus sedes de Bogotá, Pereira, Cali, Medellín, Barranquilla y Bucaramanga. 
- El área de Comunicación Corporativa de $\mathrm{ABB}$ en Colombia.

- Los estudiantes de las facultades de ingeniería eléctrica procedentes de 5 universidades ubicadas en las ciudades de Bogotá y Medellín que participaron en el programa de transferencia de conocimiento de ABB. Los estudiantes de las facultades de ingeniería abordados pertenecieron a las siguientes universidades colombianas: Universidad Distrital Francisco José de Caldas, Universidad Nacional de Medellín, Universidad de Antioquia, Universidad Pontificia Bolivariana y Universidad Militar

\section{Instrumentos de investigación e intervenciones}

\section{Revisión y análisis documental: Normas políticas y procedimientos para la comunicación de la marca $\mathrm{ABB}$ en Colombia}

Para cumplir los objetivos propuestos en la investigación, se llevó a cabo la revisión y análisis documental de las normas, políticas y procedimientos del Grupo ABB que se aplican en Colombia y que tienen relación con las estrategias de comunicación para dar a conocer la marca, el manejo de la comunicación interna y externa, las responsabilidades del área de Comunicaciones Corporativas y los grupos de interés relevantes.

Los documentos consultados fueron el Brand Book (Inside ABB 2010), las norma de Grupo: GD/CC-01A01 Roles and Responsibilities Chart of communications; GD/CC-01, corporate communications (ABB, 2011); GD/CC-02 Media relations (ABB 2010); el documento Brand positioning paper (Inside ABB, 2011); el documento Estrategia, misión y visión del negocio (Inside, ABB 2010); el documento plan de comunicación Interna $A B B$ (Inside ABB, 2010); documento Our people: Key to business success (Inside ABB, 2014).

La revisión de los documentos citados permitió determinar los principios de la marca $\mathrm{ABB}$ y la forma como el grupo desea comunicar sus mensajes ante sus stakeholders, definiendo los mensajes y los enfoques que deben ser adaptados en cada uno de los países donde opera la organización. De esta manera fue posible estructurar las bases para el diseño de una estrategia de comunicación para dar a conocer la marca e identificar las características de ésta, como variables de la investigación y permitió dar respuesta a las siguientes preguntas de investigación: ¿Qué tipo de conocimiento generan estas empresas industriales?; ¿Qué tipo de estrategias de comunicación desarrollan las empresas industriales para dar a conocer su marca?

\section{Aplicación del modelo de Mitchell, Agle y Wood para la identificación de los grupos de interés}

Con el objetivo de determinar cuáles eran los grupos de interés relevantes para ABB en Colombia, y analizar la posición que tenían los estudiantes de ingeniería eléctrica dentro de estos grupos, se implementó el modelo diseñado por Mitchell, Agle y Wood (1997) que identifica quién es y quién no es stakeholder basado en su clasificación como actores internos o externos que afectan o son afectados por los objetivos o resultados de una organización dada, en diferentes grados, en la medida en que poseen entre uno y tres atributos básicos: poder, legitimidad y urgencia. Actores que no presentaron al menos uno de estos atributos (no afectan los resultados y/o no son afectados por los resultados), no fueron tenidos en cuenta como stakeholders para la investigación. 
Programa de transferencia de conocimiento de la empresa a la universidad: una estrategia de comunicación para dar a conocer una marca industrial

Este modelo permitió identificar a los grupos de interés de $\mathrm{ABB}$ en Colombia desde la perspectiva de las estrategias de comunicación para dar a conocer la marca, como una variable de investigación para el trabajo. Así mismo, la aplicación de este modelo permitió dar respuesta a la siguiente pregunta de investigación: ¿Cuáles son los grupos de interés relevantes para una organización como $\mathrm{ABB}$ en términos de comunicación de la marca?

\section{Análisis de la calidad de la comunicación interna del área de Comunicaciones Corporativas para dar a conocer la marca ABB en los empleados}

Antes de revisar las percepciones de los empleados sobre las estrategias de comunicación de $\mathrm{ABB}$ para dar a conocer su marca y su eventual interés en la participación de estas actividades, fue necesario analizar la calidad de la comunicación interna dentro del área de Comunicaciones Corporativas y su papel en la implementación de estrategias de comunicación desarrolladas desde el interior de la compañía hacia sus grupos de interés externos.

Entendida la comunicación interna no solo como mensajes, sino actos, comportamientos de cada uno de los integrantes de la empresa que a su vez transmiten información acerca de su identidad, misión y manera de actuar con los diferentes grupos de interés. Según Trelles (2001) es el repertorio de procesos, mensajes y medios involucrados en la transmisión de información por parte de la organización.

Para el análisis de la calidad de la comunicación interna de $\mathrm{ABB}$ en Colombia se aplicó como instrumento de investigación la guía y cuestionario diseñados por Díaz de Quijano y Romeo (2011).que permite evaluar la calidad del sistema formal de la comunicación interna.

Encuesta para la identificación de la percepción que tienen los empleados respecto a la estrategia de comunicación de ABB para dar a conocer su marca

Una vez identificadas las estrategias de comunicación de ABB para dar a conocer su marca, así como la posición de los estudiantes de ingeniería eléctrica dentro de estas estrategias, fue necesario determinar la percepción de los empleados por medio de la aplicación de una encuesta estandarizada de 15 preguntas cerradas y una abierta cuyo diseño se ajustó específicamente al caso de ABB Colombia, tomando como base para las preguntas los grupos de interés identificados durante la revisión documental y la estrategia de comunicación de ABB en los años 2010, 2011 y 2012. La encuesta aplicada a los empleados como instrumento de investigación, se realizó con un muestreo no probabilístico por conveniencia tomando las respuestas de ciento cuarenta y ocho (148) empleados de las áreas de negocio de un total de 234 que recibieron la encuesta.

Para el diseño de la encuesta se tomó como referente el esquema desarrollado por Goetz y LeCompte (1998) que plantea la necesidad de presentar la hipótesis de la encuesta, sus objetivos y la determinación de las variables para estructurar las preguntas adecuadamente.

El cuestionario fue enviado a los empleados de ABB dentro de los que se encuentran hombres y mujeres de 18 a 60 años (datos confirmados con depto. de Recursos Humanos). Se indagó sobre el nivel de educación, las áreas del negocio a las que pertenecen los encuestados, y las ciudades en donde se encuentran sus oficinas: Bogotá, Cali, Barranquilla, Bucaramanga y Pereira. 
Las preguntas abarcaron el conocimiento de los empleados de ABB antes de haber trabajado en la organización, los atributos diferenciadores de la marca, los conceptos que la definen, la frecuencia de participación de los empleados en las estrategias de comunicación de la marca, la percepción que tienen frente al alcance de las estrategias de comunicación para dar a conocer su marca en los grupos de interés más relevantes y el que tienen acerca de los estudiantes de ingeniería. $\mathrm{Y}$ al mismo tiempo que se cuestionó sobre el posible interés de los empleados en participar en las estrategias de comunicación Con este instrumento, fue posible dar respuesta a la siguiente pregunta de investigación: ¿Qué tipo de empleados dentro de la organización pueden ser los transmisores de ese conocimiento?

\section{Diseño e implementación del programa de transferencia de conocimiento de ABB}

A partir de los hallazgos encontrados en la aplicación de las técnicas ya mencionadas, se planteó el diseño e implementación de una prueba piloto para el programa de transferencia de conocimiento liderado por los empleados donde los estudiantes de ingeniería eléctrica fueron los receptores de esta información. La estructura del programa de transferencia de conocimiento se basó en algunos conceptos de la metodología de marco lógico planteados por Ortegón y Prieto (2005). Al ser una herramienta diseñada para facilitar el proceso de conceptualización, diseño, ejecución y evaluación de un programa. Su énfasis está centrado en la orientación por objetivos, la orientación hacia los grupos beneficiarios $y$ en facilitar la participación y la comunicación entre las partes involucradas. También, se consideró necesario revisar con algunos miembros de las áreas de negocios y los gerentes de las mismas, cuáles eran sus ideas y necesidades para el programa de transferencia de conocimiento, las cuales contaron con la autorización de los jefes y estaban alineadas con los objetivos del negocio.

Para ello, se optó en hacer este grupo focal con una guía previamente diseñada aplicada a un gerente de una de las áreas de negocio y cuatro empleados de ABB Colombia que contestaron la encuesta de percepción de comunicación de la marca, con el fin de dar a conocer sus intereses en la implementación del programa de transferencia de conocimiento.

Los siguientes datos permitieron estructurar el programa de transferencia de conocimiento a) Características de la marca $\mathrm{ABB}$ y los mensajes que busca comunicar en sus grupos de interés externos. b) Relevancia del grupo de interés de estudiantes de ingeniería eléctrica para las estrategias de comunicación con el objetivo de dar a conocer la marca ABB. c) Tipo de conocimiento que genera una organización como $\mathrm{ABB}$ y que puede ser transmitido a sus grupos de interés externos con el objetivo de dar a conocer la marca y sus atributos diferenciadores. d) Conocimiento de los empleados de ABB frente a las características de la marca. e) Nivel de precepción de los empleados frente a las estrategias de comunicación de ABB para dar a conocer la marca. f) Revisión de los objetivos del negocio. Este instrumento permitió dar respuesta a la siguiente pregunta de investigación: ¿Cómo se debe estructurar un programa de transferencia de conocimiento para dar a conocer una marca industrial? 
Encuesta para identificar la percepción de los estudiantes como participantes del programa de transferencia de conocimiento de $\mathrm{ABB}$

Para conocer los efectos de la implementación de un programa de transferencia de conocimiento sobre las estrategias de se diseñó una encuesta que abordó a los estudiantes de ingeniería eléctrica para determinar su conocimiento previo sobre la marca $A B B$ y sus percepciones después de haber participado en el programa. Dicha encuesta se aplicó con un muestreo no probabilístico realizado por conveniencia tomando las respuestas de quinientos cincuenta y dos (552) estudiantes que respondieron a las preguntas de un total de setecientos diez y siete (717) que participaron en el programa de transferencia de conocimiento.

Entrevista sobre la percepción de un empleado de ABB que participó como transmisor de conocimiento en el programa de transferencia de conocimiento

En cuanto a la percepción de los empleados frente al programa de transferencia de conocimiento se desarrolló una entrevista semiestructurada con un cuestionario de preguntas cerradas y abiertas para dar espacio a los comentarios del entrevistado.

Hay que mencionar que este cuestionario contó con 10 preguntas que abarcaron los siguientes temas: a) Percepción del empleado de ABB como promotor de la marca acerca del programa de transferencia de conocimiento; b) Razones que motivaron al empleado a participa en este programa. Y se aplicó de manera personalizada a un empleado de $\mathrm{ABB}$ que participó como promotor en el programa de transferencia de conocimiento y la información obtenida en la entrevista fue recogida por medio de una grabación que fue transcrita al documento de la presente investigación.

La información obtenida, ayudó a conocer fuera de la percepción del empleado, sí existen otras compañías que realizan este tipo de iniciativas sumado al alcance de estos programas en aspectos como la educación y las oportunidades de negocios,

\section{Resultados}

\section{Transferencia de conocimientos en las relaciones académico empresariales}

Partiendo de lo expuesto por Zoraya (2012) sobre el concepto de transferencia de conocimiento, se determina éste como un proceso en red que agrupa personas $\mathrm{u}$ organizaciones cuya finalidad es trasladar el conocimiento adquirido por estar intimamente ligados a procesos de innovación tecnológica sumado a la experiencia adquirida a lo largo del tiempo, como es el caso ABB.

Cabe mencionar, que el obtetivo de esta transmisión de conocimiento no es solo informar y difundir, sino lograr aprovechar estos espacios para transformar a los participantes de este proceso en la búsqueda constante de este tipo de conocimiento. De esta manera logra la organización afianzar su ventaja competitiva al diferenciarse de las demás.

De igual modo, generar capital intelectual como lo determinan Chakrabarti y Santoro (2004) gracias a la formación permanente de estos procesos de aprendizaje diseñados para que los alumnos y evaluaciones de los mismos se conviertan en una garantía profesional de los conocimientos que poseen éstos, tanto para ingresar a un mundo laboral como también para promoverse dentro del mismo. 
Estas relaciones académico empresariales se constituyen como un espacio de actividades de difusión y transferencia de conocimiento que puede tener beneficios sobre las estrategias de comunicación de la marca de la empresa que propicia el espacio.

\section{Gestión del conocimiento de la empresa y la marca industrial $A B B$}

Se identificó que el tipo de conocimiento que genera la empresa multinacional suiza de ingeniería $\mathrm{ABB}$, es de carácter incorporado ya que la especificidad de sus productos y sistemas para los sectores industriales de petróleo y gas, minería y petroquímica, automatización, robótica, sector energético, entre otros, son la base del negocio.

$\mathrm{ABB}$ es una marca industrial o $\mathrm{B} 2 \mathrm{~B}$ que de acuerdo a los planteamientos de Webster (1978) desarrolla bienes y servicios ofrecidos a clientes industriales e instituciones que son los que utilizan los bienes y servicios adquiridos en su propia producción. Según la clasificación de productos para las marcas B2B que propone Pride (2006), los productos de $A B B$ se encuentran dentro de las categorías de equipos y accesorios, partes y componentes, suministros para el mantenimiento, reparación u operación y servicios asociados.

Mediante la revisión de los conceptos de marca, fue posible identificar la marca $\mathrm{ABB}$ de tipo industrial corporativa, que hace referencia a todos los elementos de la organización y a todos sus significados, que según Ollé y Riu (2009) no es solo un elemento de la empresa, es su filosofía, es la forma de concretar todos los esfuerzos empresariales, analizar la cultura $\mathrm{y}$ conectarla con el consumidor. El nombre ABB y el símbolo de su marca se utilizan para comunicarse con sus empleados, con sus clientes, con sus proveedores, con la competencia y con la sociedad en general.

\section{Estrategias de comunicación de ABB en Colombia para dar a conocer su marca en sus grupos de interés relevantes}

\section{Estudiantes universitarios como grupo de interés relevante}

Con el objetivo de identificar los grupos de interés relevantes desde la perspectiva del área de comunicaciones y la ubicación de los estudiantes universitarios como grupo, se aplicó del modelo de Mitchell, Agle y Wood (1997) que identifica quien es stakeholder dependiendo de si cumple mínimo con una de estas tres características: poder, legitimidad o urgencia. Al respecto se evidenció que el grupo de estudiantes de Ingeniería eléctrica tiene legitimidad para $\mathrm{ABB}$, en términos de deseabilidad y conveniencia en el sentido que, por tratarse de una empresa de ingeniería, requiere de fuerza laboral acorde a sus conocimientos y son los ingenieros eléctricos los futuros trabajadores de ABB. Del mismo modo, en los imperativos estratégicos definidos por $\mathrm{ABB}$, atraer a los jóvenes talentos y desarrollarlos para futuros roles de liderazgo, hace referencia a las personas que ingresarán a la organización para contribuir con su crecimiento, y estos jóvenes son los profesionales de ingeniería eléctrica que en algún momento fueron estudiantes universitarios.

Un aspecto a resaltar es que los estudiantes universitarios son catalogados por el propio grupo como los futuros clientes de productos y servicios ABB. De esta manera, fue posible corroborar que son un grupo de interés importante dentro de las estrategias de comunicación para dar a conocer la marca y dentro de la presente investigación, cobra relevancia el hecho de contemplar a este grupo como público 
Programa de transferencia de conocimiento de la empresa a la universidad: una estrategia de comunicación para dar a conocer una marca industrial

objetivo de un programa de transferencia de conocimiento.

\section{Estrategias de comunicación de ABB hacia los estudiantes universitarios}

Las actividades de comunicación de ABB en Colombia hacia los estudiantes de ingeniería se basan en la publicación de ofertas laborales en bolsas de empleo de interés para el sector, la participación en ferias universitarias e implementación del programa de prácticas empresariales. De acuerdo a revisión de la estrategia de comunicación de la marca $\mathrm{ABB}$ hacia sus grupos externos, se evidenció que del año 2010 al año 2012 no se implementó una estrategia clara para abordar de manera relevante al grupo de estudiantes/futuros clientes. La razón por la que $\mathrm{ABB}$ ha participado en ferias universitarias ha sido para tomar hojas de vida de posibles practicantes o profesionales. De este modo, se encontró se ha enfocado en la búsqueda de personal para trabajar en ABB, y no a comunicar los atributos de la marca hacia los estudiantes, futuros clientes ni muchos menos el transferir conocimiento.

De esta manera, teniendo en cuenta que el Departamento de Recursos Humanos es el encargado de programar las ferias universitarias, las ofertas laborales y las prácticas empresariales, para esta investigación se revisó toda la información sobre estas relaciones académicoempresariales y se encontró que no existe un registro del número de ofertas laborales. Referente a la participación de $\mathrm{ABB}$ en ferias universitarias, como un espacio para recoger hojas de vida, se presentan los siguientes datos.

\begin{tabular}{|c|c|c|c|}
\hline AÑO & $\begin{array}{c}\text {.Número } \\
\text { eventos, para } \\
\text { estudiantes- } \\
\text { Ferias } \\
\text { universitarias }\end{array}$ & $\begin{array}{c}\text { Número de } \\
\text { empleados } \\
\text { ABB } \\
\text { participantes } \\
\text { en los eventos }\end{array}$ & $\begin{array}{c}\text { Número de } \\
\text { asistentes en } \\
\text { base de datos } \\
\text { ABB Colombia }\end{array}$ \\
\hline 2010 & 1 & 3 & 15 \\
\hline 2011 & 2 & 6 & 50 \\
\hline 2012 & 3 & 10 & 80 \\
\hline TOTAL & $\mathbf{6}$ & $\mathbf{1 9}$ & $\mathbf{1 4 5}$ \\
\hline
\end{tabular}

Tabla 3-1 Número de actividades de comunicación de marca hacia los estudiantes futuros clientes

Fuente: Registro de actividades con estudiantes. Gerencia de Comunicaciones Corporativas ABB Colombia.

La información de la tabla revela que durante los años 2010, 2011 y 2012, ABB participó en 6 ferias universitarias para acercarse a los estudiantes de ingeniería, futuros clientes de productos y servicios de $\mathrm{ABB}$, donde participaron 19 empleados y se logró una base de datos de 145 estudiantes. Según las cifras, el promedio anual de estudiantes de ingeniería abordados en esta clase de escenarios ha sido de 48 .

De acuerdo a información suministrada por el mismo departamento, se encontró que los empleados asisten a estos eventos por solicitud directa del área de Recursos Humanos, y casi siempre son empleados que han realizado estudios en la universidad donde se lleva a cabo la feria universitaria. Con respecto a las bolsas de empleo, es el Departamento de Recursos Humanos la encargada de manejar la herramienta y ningún empleado de otras áreas se involucra en este proceso.

\section{Departamento de Comunicaciones Corporativas: líder de la estrategia de comunicación de marca del interior al exterior de la organización}

Para determinar cómo diseñar un programa de transferencia de conocimiento del interior al exterior de la organización, fue necesario medir la calidad de la 
comunicación interna con el objetivo de identificar los objetivos y flujos de comunicación que debían ser usados para diseñar e implementar el programa. Cuyo principal objetivo era que los empleados conozcan la estrategia, políticas de la empresa, resultados perseguidos, valores, principios de la marca y los objetivos del negocio, Esto significa que el programa de transferencia de conocimiento, debía ser contemplado como estratégico.

De esta manera, para lograr su fin se usaron los canales de comunicación que la organización que posee, como fueron el Noticiero interno digital Notiflash Informativo, la página web interna llamada Inside $\mathrm{ABB}$, las Carteleras Informativas, la Inducción para el personal nuevo y los videos.

Posteriormente, fue importante revisar los flujos de comunicación para cada uno de estos objetivos, y cómo se podían aplicar a este programa. Al respecto se encontró que el programa de transferencia de conocimiento debía utilizar el flujo de comunicación descendente que es el que utiliza la organización para comunicar sus objetivos estratégicos, que parten de la alta dirección y son informados hacia los niveles inferiores de la empresa lo que obliga a que los miembros de la alta gerencia estén alineados con el programa.

\section{Empleados como transmisores del conocimiento y promotores de la marca}

Mediante la consulta al Departamento de Recursos Humanos, se encontró que los empleados potenciales para participar en un programa de transferencia de conocimiento debían tener conocimientos técnicos sobre productos y servicios de $\mathrm{ABB}$, la posibilidad de asistir a las universidades, preparar los cursos y diseñar planes estratégicos. Quienes forman parte de las siguientes áreas: Metalmecánica (97), Distribución (86), Sistematización (99) y Servicio técnico (130) y a la vez hacen parte del grupo operativo que permanece en la fábrica de $\mathrm{ABB}$ en Pereira En este orden de ideas se evidenció que 234 empleados podía participar de manera activa en los programas de comunicación de la marca hacia los estudiantes de ingeniería.

Ahora bien, las personas que tienen conocimientos sobre los productos y servicios de $\mathrm{ABB}$ en Colombia son los gerentes de ventas y de producto, ingenieros técnicos comerciales, de proyectos y de servicios. De acuerdo a la información obtenida, ABB Colombia cuenta con 94 personas que tienen este perfil, pero se abordó a un grupo de personas más amplio con el fin de analizar si el enfoque hacia las personas con perfil comercial era el correcto.

Con base en lo anterior, se consideró útil preguntar a estas 234 personas sobre la percepción que tenían respecto a la estrategia de comunicación de la marca ABB y su eventual rol en ella.

\section{Percepción de los empleados sobre las estrategias de comunicación de la marca ABB en Colombia}

De acuerdo a los objetivos de la investigación, a mediados del año 2013 se implementó una encuesta estandarizada que consultó a los empleados sobre la percepción que tenían frente a las estrategias de comunicación de la marca ABB enfocadas hacia los 3 grupos de interés más relevantes para la organización en términos de comunicación externa (clientes-comunidad-estudiantes de ingeniería).

Los resultados dejaron ver que antes de trabajar en la empresa el $66 \%$ de los 
empleados encuestados si conocía la marca y el 34\% no la conocía. Para ser una organización con más de 53 años de presencia en Colombia, con operaciones en todo el país y alrededor de 700 empleados, este porcentaje de conocimiento previo es bajo.

En cuanto a la percepción de los empleados frente a las estrategias de comunicación dirigidas hacia los estudiantes de ingeniería, se evidenció que el $71 \%$ de los empleados percibían esta estrategia con muy alto impacto, lo que fue coherente con los imperativos estratégicos del grupo que hacen referencia a la importancia de atraer a los jóvenes y desarrollarlos para futuros roles de liderazgo de acuerdo al documento Our people (2011). Sin embargo, el análisis de las actividades realizadas por $\mathrm{ABB}$ Colombia durante los años 2010, 2011 y 2012, reveló que en la práctica no se han desarrollado estrategias claramente definidas ni se ha destinado tiempo o recursos económicos hacia los estudiantes de ingeniería. Esto muestra una incoherencia entre los objetivos de la organización y la realidad de la implementación de las estrategias.

Por otra parte, más del $91 \%$ de los encuestados afirmó no conocer ninguna estrategia de comunicación dirigida hacia los estudiantes de ingeniería. Al revisar la implementación de la estrategia de comunicación de ABB hacia los estudiantes durante los años 2010, 2011 y 2012 en cuanto al número de empleados participantes, se evidenció que no ha existido en Colombia un programa estructurado para la comunicación de la marca ABB hacia stakeholder y que existe desconocimiento de los empleados sobre esta estrategia.

El $87 \%$ de los empleados encuestados afirmó estar interesado en participar de las estrategias de comunicación de la marca. En este sentido se hace evidente la voluntad de los empleados de contribuir con los objetivos de la organización en términos de comunicación.

Finalmente el $54 \%$ opinó que los conocimientos más representativos para contribuir con la estrategia de comunicación de la marca $\mathrm{ABB}$, eran los conocimientos técnicos referentes a los productos $\mathrm{y}$ servicios que promueve la compañía.

\section{Diseño e implementación del programa de transferencia de conocimiento}

Por consiguiente, se planteó la creación de un programa cuyo nombre reuniera los objetivos de comunicación dirigidos a los estudiantes de ingeniería y se encontrara acorde con los principios de las relaciones académico- empresariales, donde la gestión del conocimiento juega un papel muy importante.

Seleccionando el nombre SKP (sharing Knowledge program), dicho nombre fue definido por el área de Comunicaciones Corporativas para referirse a una iniciativa que buscaba compartir los conocimientos de la organización con sus grupos de interés externos. El nombre se planteó en inglés con el objetivo de servir de ejemplo para iniciativas similares en otros países donde opera el grupo ABB, y cuyo lenguaje universal es el inglés.

A continuación se presentan las fases del programa SKP

\section{Fases del programa SKP}

Fase 1: Entrenamiento a empleados / promotores de la marca ABB. E -learning del grupo ABB.

Los empleados trasmisores del conocimiento y promotores de la marca 
debían realizar el curso virtual llamado Building our Brand (2010) disponible en la página web corporativa. Este curso describe los principios de la marca ABB $\mathrm{y}$ sus atributos diferenciadores. Todo promotor de la marca ABB debía realizar este entrenamiento que se programaba por el Departamento de Comunicaciones Corporativas.

Fase 2: Diseño de los cursos gratuitos del programa SKP

- Cursos universidades: Teniendo en cuenta los objetivos de comunicación de la compañía, cada área de negocio tuvo la oportunidad de presentar un listado de temas sobre productos y servicios de ABB que debía ser suministrado a los decanos o directores de las facultades de ingeniería de las universidades de interés, para determinar si se ajustaba a sus programas académicos y complementaba las enseñanzas impartidas. Los empleados acudieron a los directores de los programas de ingeniería de las universidades en donde estudiaron sus carreras profesionales para coordinar los cursos que se querían realizar.

- Cursos en las instalaciones de ABB: Los empleados de ABB tuvieron la oportunidad de desarrollar estos cursos.

Tras la implementación del programa SKP, a continuación se presenta en la siguiente tabla los cursos que se dictaron en entre septiembre 2013 y Octubre 2014

\begin{tabular}{|c|c|}
\hline Título del curso & 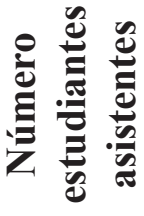 \\
\hline $\begin{array}{l}\text { Eficiencia Energética en aplicaciones con } \\
\text { máquinas rotativas }\end{array}$ & 20 \\
\hline Dimensionamiento y selección de Motores & 50 \\
\hline $\begin{array}{l}\text { Variadores de Velocidad: Principio de } \\
\text { funcionamiento y estructura básica }\end{array}$ & 98 \\
\hline Transmisión de potencia mecánica & 15 \\
\hline $\begin{array}{l}\text { "Normativa IEC y ANSI aplicable a equipos } \\
\text { eléctricos para Subestaciones" }\end{array}$ & 25 \\
\hline $\begin{array}{l}\text { Protección y automatización de sistemas } \\
\text { eléctricos industriales en baja tensión }\end{array}$ & 80 \\
\hline $\begin{array}{l}\text { Cálculos de corriente de falla y cálculo de } \\
\text { instalaciones de baja tensión. }\end{array}$ & 135 \\
\hline $\begin{array}{l}\text { Selección de dispositivos de protección contra } \\
\text { sobretensiones y coordinación de protecciones } \\
\text { en instalaciones de baja tensión }\end{array}$ & 60 \\
\hline $\begin{array}{l}\text { Eficiencia energética en cargadores eléctricos } \\
\text { de carga rápida }\end{array}$ & 85 \\
\hline Normatividad en motores NEMA & 60 \\
\hline Características de las bandas transportadoras & 89 \\
\hline $\begin{array}{l}\text { Total estudiantes que asistieron y tomaron } \\
\text { los cursos del programa SKP }\end{array}$ & 717 \\
\hline
\end{tabular}

Tabla 3-2. Listado de cursos del programa y asistentes del programa SKP. Prueba piloto Fuente: Creación propia como parte del programa SKP para la comunicación de la marca.

El programa logró abordar a 717 estudiantes de ingeniería de 5 universidades del país donde 10 empleados fueron los responsables de transferir el conocimiento.

\section{Rol del Departamento de Comunicaciones Corporativas}

La gerencia de Comunicaciones Corporativas se encargó de diseñar el plan de comunicación interna y externa del programa, así como también las piezas requeridas para difundir los cursos, su logística y garantizar la disponibilidad de material publicitario para entregar a los estudiantes que participaron en el SKP. 
Toda la información sobre los cursos y el programa SKP se publicó en los medios de comunicación internos (correo electrónico, carteleras, televisores, noticiero audiovisual y noticiero digital, Notiflash), $\mathrm{y}$ en los medios de comunicación externa como redes sociales, artículos de prensa, participación en ferias y muestras comerciales. Además, la convocatoria para asistir a estos cursos se realizó por medio de redes sociales Facebook y Twitter donde se publicó la información, las fechas, el lugar y se especificó el número de cupos disponibles.

Percepción de los estudiantes de ingeniería frente al programa de transferencia de conocimiento como estrategia de comunicación para dar a conocer la marca $\mathrm{ABB}$

Posterior a la implementación del programa de transferencia de conocimiento SKP, se realizó una encuesta de percepción aplicada a los 717 estudiantes que participaron en cada uno de los 14 cursos del programa SKP para conocer las opiniones de la implementación de este programa y su relación con las estrategias de comunicación implementadas para dar a conocer la marca.

Los resultados demostraron que el $68 \%$ de los encuestados afirmó no haber conocido la marca $\mathrm{ABB}$ antes de participar en los SKP. Este resultado evidencia la manera como los programas del tipo del SKP, pueden contribuir al conocimiento de la marca por parte de un grupo de interés no abordado previamente. La relación académico empresarial planteada por $\mathrm{ABB}$ mediante el programa de transferencia de conocimiento ofreció a la empresa la posibilidad de dar a conocer sus atributos diferenciadores, los mensajes que desea transmitir, los sectores en los que se especializa y logró mostrar la aplicación de los conocimientos de sus productos y servicios. Desde una perspectiva de la comunicación, resulta valioso que una empresa industrial especializada tenga la posibilidad de darse a conocer y permitir la identificación de los estudiantes con la marca, que en el futuro se podrán convertir en clientes.

En cuanto a la educación, les permite a los estudiantes desarrollar ciertas competencias, conocer de cerca el sector y abrirles la posibilidad de entablar relaciones, es decir crear redes, parte esencial de la transferencia del conocimiento. Esto se refleja porque el $87 \%$ de los encuestados consideró que los cursos del programa SKP contribuyen a mejorar la calidad de la educación. En este punto, vale la pena retomar los planteamientos de Casas y Luna (1997) quienes consideran a las relaciones académico empresariales como un beneficio mutuo en términos de gestión del conocimiento para el desarrollo económico de las sociedades, y de esta manera la información que $\mathrm{ABB}$ puede compartir a través del programa de transferencia del conocimiento le ayudará a los estudiantes en su formación académica que podrá tener repercusiones en su futuro desempeño laboral.

Por otro lado, se encontró que el 93\% de los encuestados consideró que estos programas de transferencia de conocimiento contribuyen al conocimiento de la marca en los estudiantes, futuros clientes. Estos resultados validan por completo el planteamiento de esta investigación donde este tipo de programas pueden contribuir a que la marca sea conocida por sus grupos de interés externos y permiten dimensionar los efectos de una iniciativa de este este tipo dentro de las estrategias de comunicación de la marca.

Se subraya que el $92 \%$ de los encuestados mostró interés en trabajar en ABB luego de haber conocido la marca por primera vez, 
sus atributos y mensajes diferenciadores, no solo valoran los cursos SKP para mejorar la calidad de la educación y dar a conocer la marca, sino que también les gustaría hacer parte de la organización. Este es sin duda uno de los resultados más significativos para la presente investigación ya que generan una conexión entre los estudiantes y la empresa basada en las aspiraciones laborales de los estudiantes.

Finalmente, el $86 \%$ de los encuestados manifestó que si pudiera hacerlo, compraría productos y soluciones de $\mathrm{ABB}$ en el futuro. Esto demuestra aunque los estudiantes de ingeniería no puedan comprar productos o servicios de $\mathrm{ABB}$ de manera inmediata y directa, por el tipo de negocio que es, por sus precios y porque es una compañía $\mathrm{B} 2 \mathrm{~B}$, pueden en una posibilidad futura convertirse en clientes a largo plazo.

\section{Percepción de los empleados promotores de la marca acerca del programa de transferencia de conocimiento}

Al ser esencial conocer la opinión de los empleados que participaron como transmisores y promotores de la marca en el programa de transferencia de conocimiento SKP, se realizó una entrevista a uno de ellos y debido a sus resultados demostró que el programa de transferencia de conocimiento, fue bien recibido quien lo catalogó como una buena estrategia de comunicación para dar a conocer la marca $\mathrm{y}$ transferir conocimiento.

\section{Discusión de resultados}

La presente investigación ha permitido entender la importancia de la gestión del conocimiento en las relaciones académicoempresariales. Del mismo modo establecer fases planteadas por Wiig (1997) como, la generación, codificación, transferencia y su utilización, aportando a la definición de los actores que se involucran en este proceso.
Siendo la empresa la encargada de generar, codificar y transferir este conocimiento y la academia es la de utilizarlo para contribuir al desarrollo de la sociedad.

De esta manera, los empleados como miembros de la empresa están en la capacidad de codificar la información y transferirla a los estudiantes como se logró en el programa de transferencia de conocimiento llamado SKP (Sharing knowledge program) liderado por el Departamento de Comunicaciones Corporativas al fortalecer las relaciones académico-empresariales entre ABB y las facultades de ingeniería, contribuyendo así a la estrategia de comunicación planteada.

Por otro lado, se identificó a los empleados de ABB como promotores de la marca y transmisores del conocimiento, siendo los perfiles ideales para este programa los de tienen conocimientos comerciales y técnicos sobre los productos o servicios de la empresa, por estar mejor capacitados. Cabe destacar, la necesidad de reforzar el conocimiento de las estrategias aplicadas hacia sus grupos de interés más relevantes como los clientes.

De igual maner, el programa permitió reforzar los conceptos de la marca junto con los mensajes que desea dar a conocer en los empleados elegidos para tal fin y la definición de los cursos que hicieron parte de la prueba piloto, que fueron en total 14 , liderados por 10 empleados impactando en 717 estudiantes de ingeniería eléctrica de 5 universidades de Bogotá y Medellín.

Por lo cual, la aplicación de una encuesta de satisfacción sobre los cursos en mención era esencial hacerla. Dando como resultado que el $70 \%$ fue capaz de identificar todos los mensajes con los ABB buscaba comunicar los atributos de su marca. El $64 \%$ logró identificar el slogan de ABB lo 
que permitió crear confianza con la marca y tener una mayor cercanía. El 81\% identificó los años que lleva $\mathrm{ABB}$ en el mundo, un $67 \%$ logró identificar los sectores en los que se aplican las tecnologías, productos y servicios de la empresa. E1 90\% identificó de forma acertada los años que lleva ABB en Colombia, logrando así dimensionar la trayectoria de la organización a nivel nacional e internacional.

En relación a la mejora de la calidad de la educación, un $87 \%$ lo considera como positivo. Esto se logra al afianzar las relaciones académico empresarial, reflejado en los cursos que fueron espacios no solo de conocimiento sino una manera práctica de entender el mundo que les espera.

Por otra parte, la entrevista realizada al empleado que participó como transmisor y promotor de la marca $\mathrm{ABB}$ en el programa SKP evidenció que los empleados de $\mathrm{ABB}$ pueden aportar sus conocimientos para dar a conocer una marca industrial como la de ABB. Destacando la frase que utilizó el entrevistado para describir el programa, "esta estrategia es como una escopeta de perdigón que con un solo tiro dispara a muchos frentes", que valida el planteamiento de Wiig (1997) quien entiende el proceso de gestión de conocimiento dentro de la empresa como la generación, la codificación, la transferencia y la utilización. Así, se entiende entonces que la empresa, generadora del conocimiento, es la escopeta de perdigón, las balas son el conocimiento y los estudiantes, son quienes utilizan este conocimiento para su beneficio.

Finalmente, las respuestas del empleado entrevistado validaron la hipótesis planteada para la entrevista ya que se evidencia que para este empleado de ABB el programa de transferencia de conocimiento ha contribuido a la estrategia de comunicación de la organización para dar a conocer su marca en los estudiantes, futuros clientes.

\section{Conclusiones y recomendaciones}

El análisis de los objetivos de negocio, la relevancia de los estudiantes universitarios como grupo de interés relevante, el interés de los empleados de participar en las estrategias de comunicación para dar a conocer la marca y los resultados de la encuesta aplicada a los estudiantes que participaron en el programa de transferencia de conocimiento de $\mathrm{ABB}$, permitieron validar laidea presentada en la investigación de que un programa de transferencia de conocimiento estructurado, que involucre a la organización para definir los mensajes y que seleccione adecuadamente a sus grupos de interés, puede contribuir al conocimiento o refuerzo de los aspectos diferenciadores de la marca ABB ante los estudiantes de ingeniería, aportando al mejoramiento de las estrategias de comunicación de la organización.

El programa de transferencia de conocimiento SKP desarrollado en esta investigación, claramente aportó a la estrategia de comunicación para dar a conocer la marca en términos de número de estudiantes abordados, número de empleados participantes y nivel de involucramiento por parte de la Gerencia de Comunicaciones Corporativas de ABB

Los efectos de la implementación de la prueba piloto del programa SKP (717 estudiantes abordados, involucramiento de empleados y liderazgo del área de comunicaciones) se deben en parte a la forma como se difundió el programa al interior de la organización mediante piezas informativas publicadas en todos los medios disponibles. La difusión de esta 
iniciativa en el fan page de Facebook, como herramienta de comunicación externa, permitió generar una interacción constante con los estudiantes que participaron en el programa y con aquellos que deseaban participar, de esta manera el programa de trasferencia de conocimiento no se limitó a los cursos dictados personalmente, sino que trascendió a programas virtuales con mayor alcance, y fue más allá de los comentarios en redes sociales y de las notas de prensa nacional.

De esta manera, se considera que para lograr efectos significativos sobre la estrategia de comunicación de la marca, mediante la implementación de un programa de transferencia de conocimiento, es necesario contar con el involucramiento de la alta gerencia de la organización, definir los mensajes correctamente, identificar los canales de comunicación más apropiados e involucrar a los empleados como transmisores. También es importante tener presente que por más estructurado que esté el programa de comunicación de la marca, si los gerentes y/o tomadores de decisiones dentro de la organización, no consideran que este tenga un impacto en los objetivos de sus áreas, no tendrá éxito.

Si se hace un análisis del potencial que tiene el programa SKP en ABB Colombia, en términos de número de estudiantes de ingeniería que pueden ser abordados, se pensaría entonces que si en un año se logró abordar a 717 estudiantes de ingeniería, mediante la realización de 14 cursos ( un promedio de 51 estudiantes por curso) promovidos por 10 empleados, en este mismo periodo, si cada uno de los 94 empleados de ABB con cargos comerciales y conocimientos técnicos dictan solamente un curso durante el año, la marca podría darse a conocer en 4.794 estudiantes. Este número sumado al compromiso de 94 empleados como promotores de la marca y a la exposición en medios de comunicación, generaría un gran impacto para la marca ABB.

Por otro lado, sería interesante analizar a futuro si programas de transferencia de conocimiento como el SKP, mediante el liderazgo de un proceso de comunicación estratégica a cargo del área de Comunicaciones Corporativas, son aplicables a otro tipo de organizaciones que busquen dar a conocer sus marcas. Quizá las organizaciones que valoran los aportes de esta área, y la consideran fundamental en la consecución de los objetivos de la organización, estén en la capacidad de estructurar un programa similar, enfocado a sus necesidades particulares de comunicación de marca.

\section{Referencia Bibliográfica}

Aaker, D. (2002). Construir marcas poderosas. Barcelona: Editorial Gestión 2000.

Ames, B. (1968). Marketing planning for industrial products. Harvard Business Review, $44(10), 100-111$.

Andrade, H. (2005). Comunicación organizacional interna: proceso, disciplina y técnica. México: Editorial Netbiblo.

Andreu, R. y Sieber, S. (1999). La gestión integral del conocimiento y del aprendizaje. En: Revista Economía Industrial, 326(3), 113120.

Aubel, J. (1994). Guidelines for studies using the group interview technique. En: International Labour Organization, 68, 34-67.

Bekin, S. F. (1995). Conversando sobre endomarketing. São Paulo: Makron Books.

Blankson, C. y Kalafatis, S. (2001). The development of a consumer/customer- derived generic typology of positioning strategies. En: Marketing Theory and Practice Journal, 50 (7), $35-53$. 
Bradley, F. (1995). Marketing Management: Providing, communicating and delivering value. London: Editorial Prentice Hall.

Bravo, G. y Diaz, M. (2009). Cátedra empresarial Silicon Graphics: Un ejemplo de colaboración Universidad - Empresa. En: Revista de Ingeniería Uniandes, 27, 55-67.

Brown, J. y Duguid, P. (1991). Organizational learning and communities-of-practice: Toward a unified view of working, learning, and innovating. En: Organization science Review, 2(3), 40-57.

Buskirk, R. (1961). Principles of marketing: The management view. New York: Holt, Rinehart, and Winston, Editorial Inc.

Casas, R. y Luna, M. (1997). Gobierno, academia y empresas en México: Hacia una nueva configuración de relaciones. México: Plaza y Valdés Editores S. A.

Chakrabarti, A. y Santoro, M. (2004). Building social capital and learning environment in university - industry relationships. En: Learning and Intellectual Capital Journal, 1, 19-36.

Chiavenato, I. y Villamizar, G. (2002). Gestión del talento humano. Bogotá: McGraw-Hill.

Collado, C. (2005). La comunicación en las organizaciones. Madrid: Editorial Trillas.

Cooke, E. (1986). What is business and industrial marketing? En: Journal of Business y Industrial Marketing, 1(1), 9-17.

Inside Facebook (2009). "The Facebook Global Monitor". [Versión digital]. Consultado el 16 de septiembre de 2014, http://www. insidefacebook.com/

Costa, J. (2004). La imagen de marca, un fenómeno social. Barcelona: Editorial Paidós.

Cubillo, J. (2011). Descifrando el ADN de las marcas. Elementos esenciales para la Dirección Estratégica de Marcas. Madrid: Editorial ESIC.

Dasgupta, P. y David, P. (1992). Toward a new economics of science, Center for Economic Policy Research Conference on the Role of Technology in Economics. Palo Alto, California: Stanford University Press.
Davenport, T. y Prusak, L. (1998). Working Knowledge: How organizations manage what they know. En: Harvard Business School Press, 301(4), 105-120.

Davis, S. (2002). La Marca: máximo valor de su empresa. México: Ediciones Pearson Educación.

Díaz, J. y Losada, C. (2004). Gestión de la comunicación en las organizaciones. Barcelona: Ediciones Ariel.

Folgueiras, P. y Martínez, M. (2009). El desarrollo de competencias en la universidad a través del Aprendizaje y el Servicio Solidario. En: Revista Interamericana de Educación para la Democracia (RIED), 2(1) 55-76.

Galeano, M. (2004). Diseño de proyectos en la investigación cualitativa. Medellín: Fondo editorial universidad EAFIT.

Grant, R. (2000). Shifts in the World Economy: the Drivers of Knowledge Management. En: C Despres and D Chauvel Knowledge Horizons, 5(2), 27-53.

Goetz, J. y LeCompte, M. (1998). Etnografía y Diseño Cualitativo en Investigación Educativa. España: Editorial Morata.

Gould, B. (1997). Vinculación UniversidadSector Productivo. Una reflexión sobre la planeación y operación de programas de vinculación. En: ANUIES, 42(5), 67-100.

Grönroos, C. (1995). Relationship marketing: the strategy continuum. En: Journal of the Academy of Marketing Science, 23(4), 252254.

Gummesson, E. (1987). The new marketingdeveloping long-term interactive relationships. En: Long range planning, 20(4), 10-19.

Hatch, M. y Schultz, M. (1997). Relations between organizational culture, identity and image. En: European Journal of marketing, 31(6), 356-365. 
Hofstede, F.; Steenkamp, J.; Wedel, M. (1999). International market segmentation based on consumer-product relations. En: Journal of Marketing Research, 40(5), 1-17.

Holland, B. (1989). Motivation and job satisfaction. En: Journal of Property Management, 54(5), 32.

Hooley, G.; Broderick, A.; Möller, K. (1998). Competitive positioning and the resourcebased view of the firm. En: Journal of Strategic Marketing, 6(2), 97-116.

Karlan, D.; McConnell, M.; Mullainathan, S.; Zinman, J. (2010). Getting to the top of mind: How reminders increase saving. Boston: National Bureau of Economic Research.

Keller, K. (2003). Brand synthesis: The multidimensionality of brand knowledge. En: Journal of consumer research, 29(4), 595-600.

Kotler, P. y Solis, E. (1996). Dirección de mercadotecnia. New Jersey: Pearson Prentice Hall.

Knigth, J. (2011). Five Myths about Internationalization. En: International Higher Education, 62 (4), 14-15.

Kogut, B. y Zander, U. (1992). Knowledge of the firm: combinative capabilities, and the replication of Technology. En: Organization Science Journal, 3 (3), 383-397.

Kotler, P. (1989). From mass marketing to mass customization. En: Strategy y Leadership Journal, 17(5), 10-47.

Kotler, P. (2000). Marketing Management. New Jersey: Prentice Hall.

Lambin J. (1991). Marketing Estratégico. Madrid: Editorial McGraw-Hill.

Levitt, T. (2006). Miopía de marketing. En: Harvard Business Review, 82(7), 88-99.

Leydesdorff, L. y Meyer M. (2006). Triple Helix indicators of knowledge-based innovation systems, Introduction to the special issue. En: Research Policy, 35(10), 20-129.
Lundvall, B. (Ed.). (2010). National systems of innovation: Toward a theory of innovation and interactive learning. London: Anthem Press.

Luhmann, N. (1995). Social systems. Palo Alto, California: Stanford University Press.

Martin, A. (1993). Marketing. Barcelona: Ediciones Ariel.

Mc Alexander, J.; Schouten, J.; Koenig, H. (2002). Building brand community. En: The Journal of Marketing, 53(5), 38-54.

Mc Carthy, E. (1960). Basic marketing: a managerial approach. Homewood, Illinois: Richard D. Irwin Inc.

Mc Clelland, D. C. (1967). Achieving society. New York: Simon and Schuster; Harper Collins Publishers New Zealand Distributor.

Mc Kenna, R. (1993). Relationship Marketing: Successful strategies for the age of the costumer. Boston: Addison-Wesley.

Nonaka, I. y Taekeuchi, H. (1995). The Knowledge-Creating Company. Oxford University Press.

O' Really (2009). Asia continues to be Facebook' strongest growth region. California State University-Fullerton.

Orsenigo, L. (1989). Academic/company research programs. New York: St Martin's Press.

Ortegón, E. y Prieto, A. (2005). Metodología del marco lógico para la planificación, el seguimiento y la evaluación de proyectos y programas. Santiago de Chile: United Nations Publications.

Pizzolante N. (1993). Ingeniería de la imagen. Caracas: Editorial UCAB.

Preciado, A. (2011). Las conversaciones en la organización. Culturas organizacionales y comunicación de la cultura. En: Ponencia presentada en el congreso "Las conversaciones de la organización", organizado por la Colegiatura Colombiana en Medellín. 
Programa de transferencia de conocimiento de la empresa a la universidad: una estrategia de comunicación para dar a conocer una marca industrial

Pride, W. y Ferrell, O. (2006). Marketing: Concepts and Strategies I. Boston: Houghton Mifflin Company.

Rayburn, L. (1986). Accounting principles and marketing models related to deferred marketing costs. En: European Journal of Marketing, 20(1), 75-82.

Reichheld, F. (2003). El único número que necesita mejorar. En: Revista Harvard Business Review, 95(5), 38-46.

Rodríguez, G. (2009). The retention of efficient employees: strategic importance of employee's fidelity. En: Journal of Good Conscience, 4(2), 145-159.

Rodríguez G.; Gil F.; García J. (1999). Metodología de la investigación cualitativa. Málaga: Editorial Aljibe.

Rodríguez, D. y Opazo, M. (2007). La Comunicación de la Organización. Santiago: Universidad Católica de Chile.

Rosenbaum E.; Percy, L.; Pervan, S. (2011). Strategic brand management. New York: Oxford University.

Smith, A. y Zeithaml, C. (1999). The Intervening Hand: Contemporary International Expansion Process of the Regional Bell Operating Companies. En: Journal of Management Inquiry, 8, 34-64.

Sáez, P., y de Castro, G. (2003). Bases conceptuales para la Dirección del Conocimiento en las Organizaciones. En: Revista Madrid, 20(4), 108-129.

Stanton, W. (1964). Fundamentals of Marketing. New York: McGraw-Hill.

Tourish, D., y Hargie, O. (Eds.). (2004). Key issues in organizational communication. London: Psychology Press.

Trelles, I. (2001). Comunicación organizacional. La Habana: Ediciones Félix Varela.

Trout, J. y Rivkin, S. (1996). El nuevo posicionamiento. México: Ediciones Limusa.

Ries, A. y Trout, J. (2002). Posicionamiento: la batalla por su mente. México: McGraw-Hill.
Urban, G. (1975). Perceptor: A model for product positioning. En: Management Science Journal, 21(8), 858-871.

Van Riel, C. (1997). Comunicación corporativa. Madrid: Prentice Hall.

Varela, G. (1997). Los patrones de vinculación Universidad - Empresa en EEUU y Canadá, y sus implicaciones para América latina. En: Industrial marketing strategy, 3, 13-41.

Weisinger, H. (2001). La inteligencia emocional en el trabajo. Buenos Aires: Javier Vergara Editor.

Wensley, A.; Verwijk A.; O’Sullivan, A. (2000). Tools of Knowledge Management. En: Despres y Chauvel, 30(3), 113-133.

Winer, R. y Moore, W. (1989). Evaluating the effects of marketing-mix variables on brand positioning. En: Journal of Advertising Research, 29(1), 39-45.

Yin, R. (1999). Enhancing the quality of case studies in health services research. En: Health services research Journal, 34(5), 120-149

\section{Notas al lector}

1 Disruptivo: Es un negocio cuyas innovaciones obligaron a otras empresas a modificar su rumbo estratégico. La lista es recompilada por los editores de MIT Technology Review, que buscan las empresas que durante el año anterior hayan demostrado una tecnología original y valiosa para un mercado a gran escala y qué influya directamente en sus competidores. Después de años de investigación, $\mathrm{ABB}$ ha desarrollado el primer interruptor para corriente continua de alto voltaje (HVDC, por sus siglas en inglés) del mundo. Este interruptor combina mecanismos muy veloces con electrónica de potencia y será capaz de interrumpir en 5 milisegundos flujos de energía equivalentes al producido por una gran estación de energía - este tiempo es 30 veces más rápido que el pestañeo del ojo humano. 